\title{
Alternativas para el manejo agroecológico de especies leñosas arbustivas en agroecosistemas ganaderos del noroeste de Córdoba, Argentina
}

\author{
Reinoso Pérez, M., Joseau, M. J. y Valdez, H. A.
}

\begin{abstract}
RESUMEN
La investigación tuvo como objetivo identificar alternativas de manejo agroecológico de especies arbustivas en agroecosistemas ganaderos del noroeste de Córdoba, Argentina. Mediante técnicas participativas se determinó la dominancia de arbustivas, la importancia de los impactos atribuidos a ellas, la viabilidad de los tratamientos convencionales de control y la prefactibilidad de las alternativas de manejo agroecológico de estas. Vachellia caven, Celtis chichape, Senegalia gilliesii, Vachellia astringens, Geoffroea decorticans y Larrea divaricata fueron las especies más dominantes. El manejo, la rentabilidad, la colonización y la accesibilidad fueron los impactos negativos de mayor importancia mencionados por los actores "productivistas", mientras que para los "conservacionistas" lo fueron en sentido positivo la protección, la diversidad y la alimentación. La viabilidad de los tratamientos de control osciló entre 0,3 y 0,6, con valores más altos para rolado, rastra y la combinación de ambos. El 83,3 \% de las alternativas evaluadas obtuvo más del $50 \%$ del valor máximo posible. La presencia de múltiples atributos beneficiosos en estas especies propicia la implementación de estrategias de manejo y uso productivo en sistemas agroforestales. El pago por servicios ambientales, la poda de elevación de copa, el pastoreo rotativo secuencial vacuno-caprino, y la comercialización de los productos derivados de estas plantas, son las alternativas más promisorias.
\end{abstract}

Palabras clave: Arbustos leñosos, agroecosistemas ganaderos, manejo agroecológico.

Reinoso Pérez, M., Joseau, M. J. and Valdez, H. A. 2019. Alternatives for the agroecological management of woody shrub species in livestock agroecosystems in the northwest of Córdoba, Argentina. Agriscientia 36: 1-14

\section{SUMMARY}

The objective of this research was to identify alternatives for the agroecological management of shrub species in livestock agroecosystems in the northwest 
of Córdoba, Argentina. Participatory techniques were used to determine the dominance of shrubs, the importance of the impact attributed to them, the feasibility of conventional control treatments and the prefeasibility of the alternatives for their agroecological management. The most dominant species were Vachellia caven, Celtis chichape, Senegalia gilliesii, Vachellia astringens, Geoffroea decorticans and Larrea divaricata. Management, profitability, colonization and accessibility were the most important negative impacts for 'productive' actors, while protection, diversity and feeding weres the most relevant positive impacts according to 'conservationists'. The feasibility of the control treatments ranged between 0.3 and 0.6 , with higher values for rolling, harrowing and the combination of both. $83.3 \%$ of the agro-ecological management alternatives obtained more than $50 \%$ of the maximum possible value. The existence of multiples beneficial attributes in these species facilitates the implementation of management strategies and the productive use in agroforestal systems. The payment for environmental services, the pruning of the crown elevation, the rotational sequential grazing of cattle and goats, and the commercialization of the products derived from these plants, are the most promising alternatives.

Key words: Woody shrubs, livestock agroecosystems, agro-ecological management.

Reinoso Pérez, M.: Universidad Central "Marta Abreu" de Las Villas, Facultad de Ciencias Agropecuarias, Carretera a Camajuaní Km 5.5, CP 54830, Santa Clara, Cuba. Joseau, M. J.y Valdez, H. A.: Universidad Nacional de Córdoba, Facultad de Ciencias Agropecuarias (FCA-UNC), Félix Marrone 746, Ciudad Universitaria, CC 509, CP 5000,Córdoba, Argentina. Correspondencia a: mariorp@uclv.cu

\section{INTRODUCCIÓN}

En los últimos 150 años la combinación de procesos naturales como las sequías estacionarias con los incendios y la tala indiscriminada de recursos forestales madereros, así como el sobre o sub pastoreo, han propiciado la presencia creciente de especies arbustivas leñosas en detrimento de otras especies de interés forrajero para la ganadería, lo que ha devenido en una temática muy compleja y polémica en varias regiones del mundo, y especialmente en las regiones áridas y semiáridas (Skarpe, 1990; Archer, 1995)como partes de la región chaqueña de Argentina.

Este fenómeno, conocido como "lignificación o arbustización" (Renolfi, 2006), ha sido y es percibido por los actores sociales desde perspectivas muy contrastantes e incompatibles, que van desde una "visión erradicadora" al considerarlas como una plaga, hasta una "visión integradora" donde se revalorizan sus funciones ecosistémicas (Ledesma y Tomsic, 2011). Por esta razón no ha sido posible construir un enfoque consensuado entre productores, técnicos, académicos, investigadores y tomadores de decisiones, que detenga la homogeneización indeseable del paisaje y la pérdida de la biodiversidad en los agroecosistemas productivos en los que se encuentren estas plantas.

En el noroeste de la provincia de Córdoba, donde la ganadería vacuna y caprina dedicada a la producción de carne tiene una participación importante en la economía provincial y de Argentina (Daza y Sánchez, 2009), predominan los sistemas extensivos y semintensivos sobre pastizales y bosques degradados, con proliferación de especies leñosas arbustivas a expensas del crecimiento de las pasturas nativas e implantadas, que limitan severamente la oferta forrajera (Mussari, 2005; Kunst, 2006) y la capacidad de carga animal de los sistemas (Orellana, 2003). Esta situación ha conducido al empleo de diversos métodos de control o erradicación ya sean manuales, mecánicos, biológicos, químicos o el uso del fuego (Kunst et al., 2003; Adema, Buschiazzo, Babinec, Rucci y Gómez-Hermida, 2004; Tolozano, Pisani y Puricelli, 2016), sin la obtención de los resultados deseados por los productores ya que en general 
solo se elimina la parte aérea de las plantas; la durabilidad del tratamiento en cuestión es limitada, muchos de ellos demandan alto consumo de recursos, y finalmente el rebrote es más vigoroso contribuyendo a potenciar la "vocación leñosa" de estos agroecosistemas (Renolfi, 2006).

El objetivo de la presente investigación fue identificar alternativas para el manejo agroecológico de las especies leñosas arbustivas presentes en los agroecosistemas ganaderos del noroeste de Córdoba, Argentina.

\section{MATERIALES Y MÉTODOS}

\section{Ubicación del área de estudio}

El área de estudio se encuentra en la provincia

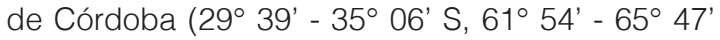
O), comprende parte de las regiones del Chaco Árido y Semiárido de la provincia fitogeográfica Chaqueña (Cabrera, 1994) y abarca las zonas agroeconómicas homogéneas (ZAH) XI-D Cruz del Eje -Zona Árida de Traslasierra de Producción Ganadera Extensiva-y XI-E Cosquín-Zona Serrana de Producción Ganadera Extensiva- descriptas por Daza y Sánchez (2009). Ambas ZAH son altitudinalmente muy variables, llegando hasta los 1800 m s. n. m. , y poseen una pluviometría con rango de distribución entre $550 \mathrm{~mm}$ al oeste y 650$700 \mathrm{~mm}$ al este.

\section{Agroecosistemas de referencia}

La investigación involucró 120 campos ganaderos dedicados a la producción de carne vacuna o caprina, ubicados en las localidades de Deán Funes, Ischilín, Avellaneda, Copacabana, Cañada de Río Pinto, Chuña, Las Canteras, El Bañado, San José de las Salinas, Huascha, Paraje El Portillo, Las Chacras, San Pedro de Toyos y Quilino (Departamento Ischilín), San Francisco del Chañar (Departamento Sobremonte), Bella Vista, Iglesia Vieja, Cruz de Caña, Paraje Yeguas Muertas, Bañado de Soto y Corrales Viejos (Departamento Cruz del Eje), Villa Tulumba, Sebastián El Cano, El Chipitín y Santa Cruz (Departamento Tulumba), San Carlos Minas, Cruz de Silva y Guasapampa (Departamento Minas) abarcando de manera indistinta las $\mathrm{ZAH}$ antes mencionadas.

\section{Metodología general de investigación}

Se siguieron los fundamentos metodológicos y procedimentales de la Investigación-Acción-
Participación recomendados por Mettrick (1993), Geilfus (2002) y Borrás et al. (2017). Bajo estas premisas se utilizaron diversas técnicas de diálogo, observación y dinámica de grupos (entrevistas semiestructuradas y abiertas, tanto individuales como grupales; observación participante y lluvia de ideas), así como herramientas participativas matriciales diseñadas para relevar la percepción de los actores sociales vinculados directa o indirectamente con el tema objeto de estudio, las que fueron aplicadas de manera secuencial en 12 talleres de intercambios de saberes y experiencias convocados para tal fin.

\section{Variables determinadas}

- Dominancia relativa de las especies arbustivas: proporción entre el puntaje obtenido según la percepción de los productores y el valor máximo posible $(10 \times n)$.

- Frecuencia de aparición de las especies arbustivas: proporción entre la frecuencia absoluta con que cada especie fue mencionada por los productores y el número total de productores participantes en el estudio ( $\mathrm{n}=$ 120).

- Importancia relativa de los impactos atribuidos a las especies arbustivas: proporción entre la sumatoria de la frecuencia de aparición del impacto en la comparación por pares y la frecuencia acumulada de aparición máxima posible $(7 \times n)$. Dada la existencia de percepciones divergentes respecto a esta variable, los entrevistados fueron agrupados según la importancia que le concedieron en sus respuestas al carácter positivo o negativo de los impactos sobre los recursos naturales (conservacionistas) y la productividad del establecimiento ganadero (productivistas), independientemente del sector ocupacional a que pertenecen.

- Viabilidad de los tratamientos empleados para el control de las especies arbustivas: proporción entre la sumatoria de los puntajes obtenidos para cada tratamiento y el valor máximo posible considerando el número de actores que participaron en la evaluación del tratamiento en cuestión $(16 \times n)$.

- Factibilidad relativa, ex-ante, de las opciones ecoamigables promisorias para propiciar el manejo agroecológico de las especies arbustivas: proporción entre la sumatoria de los puntajes obtenidos para cada opción y el valor máximo posible considerando el número de 
actores que participaron en la evaluación de la opción en cuestión (14 x n).

\section{Procesamiento estadístico de los datos primarios}

Asociada a cada variable se calcularon los estadígrafos descriptivos: media, desviación estándar, error estándar, moda y coeficiente de variación, utilizando para ello el software estadístico Infostat (Di Rienzo et al., 2017).

\section{RESULTADOS Y DISCUSIÓN}

Los resultados derivados de la aplicación de la matriz de jerarquización de dominancia o abundancia de las arbustivas (Tabla 1) en los campos ganaderos donde están presentes indican que V. caven (espinillo), C. chichape (talilla), S. gilliesii (garabato macho), $V$. astringens (garabato negro), $G$. decorticans (chañar) y $L$. divaricata (jarilla) figuraron entre las seis especies con mayor índice de dominancia o abundancia, según la percepción de los ganaderos entrevistados.
Dentro de las especies arbustivas identificadas, se encontró $G$. triacanthos L. (acacia negra), de origen exótico incluida en la base de datos de árboles y arbustos invasores de la provincia de Córdoba (Giorgisi y Tecco, 2014), sin embargo mostró una dominancia relativa baja $(0,01)$.

La literatura científica consultada aborda estas especies arbustivas desde diversas perspectivas, algunas coincidentes y otras contrapuestas, lo cual confirma la naturaleza multidimensional del tema objeto de estudio. No obstante, existe consenso sobre las características o propiedades generales que distinguen a la mayoría de ellas, a saber:

1. Elevada capacidad de adaptación y persistencia ante disturbios o condiciones adversas tales como la salinidad, la herbivoría intensa e incendios, entre otras (Bond y Midgley, 2001; Barchuk, Campos, Oviedo y Díaz, 2006), propiedad que está presente en muchas plantas leñosas de las familias Fabaceae y Zygophyllaceae a las que pertenecen la mayoría de los arbustos formadores de fachinales en el norte de Córdoba. Por ello se les confiere gran importancia ecológica como bioindicadoras de

Tabla 1. Orden de dominancia de las especies arbustivas presentes en los agroecosistemas ganaderos del noroeste de Córdoba ( $\mathrm{n}=$ 120).

\begin{tabular}{|c|c|c|c|c|}
\hline Nombre científico & Nombre vulgar & $\begin{array}{l}\text { Puntaje } \\
\text { obtenido }\end{array}$ & $\begin{array}{l}\text { Dominancia } \\
\text { relativa }\end{array}$ & $\begin{array}{l}\text { Frecuencia de } \\
\text { aparición }\end{array}$ \\
\hline Vachellia caven (Molina) Seigler \& Ebinger & espinillo, churqui, aromito & 527 & 0,44 & 0,47 \\
\hline Celtis chichape (Wedd.) Miq & talilla, tala-churqui & 226 & 0,19 & 0,27 \\
\hline Senegalia gilliesii (Steud.) Seigler \& Ebinger & garabato macho & 224 & 0,19 & 0,23 \\
\hline Vachellia astringens (Gillies ex Hook. \& Arn.) Speg. & $\begin{array}{l}\text { garabato negro, aromo } \\
\text { negro }\end{array}$ & 148 & 0,12 & 0,16 \\
\hline Geoffroea decorticans (Hook. \& Arn.) Burkart & chañar & 142 & 0,12 & 0,20 \\
\hline Larrea divaricata Cav. 1800 & jarilla & 135 & 0,11 & 0,16 \\
\hline Condalia microphylla Cav. 1799 & piquillín & 91 & 0,08 & 0,13 \\
\hline $\begin{array}{l}\text { Vachellia aroma (Gillies ex Hook. \& Arn.) Seigler \& } \\
\text { Ebinger }\end{array}$ & tusca & 69 & 0,06 & 0,08 \\
\hline Flourensia oolepis S.F. Blake y Baccharis sp. & chilcas & 56 & 0,05 & 0,07 \\
\hline Senegalia praecox (Griseb) Seigler \& Ebinger & $\begin{array}{l}\text { garabato hembra, uña } \\
\text { de gato }\end{array}$ & 55 & 0,05 & 0,07 \\
\hline Lippia turbinata Griseb. & poleo & 39 & 0,03 & 0,06 \\
\hline Trithrinax campestris (Burmeist.) Drude \& Griseb & palma caranday & 32 & 0,03 & 0,03 \\
\hline Cestrum parqui L'Hér. & duraznillo negro & 22 & 0,02 & 0,03 \\
\hline Aloysia gratissima (Gill. et Hook) Tronc. & palo amarillo & 17 & 0,01 & 0,03 \\
\hline Mimozyganthus carinatus (Griseb.) Burkart & Lata & 15 & 0,01 & 0,02 \\
\hline Cercidium praecox (Ruiz \& Pav. Ex Hook.) Harms & brea & 10 & 0,01 & 0,02 \\
\hline Gleditsia triacanthos L. & acacia negra & 10 & 0,01 & 0,01 \\
\hline Ligustrum lucidum W.T. Aiton & siempreverde & 7 & 0,01 & 0,01 \\
\hline Colletia spinosissima J.F. Gmael & barba de tigre & 5 & 0,00 & 0,01 \\
\hline Puntaje máximo posible & & 1200 & -- & -- \\
\hline
\end{tabular}


zonas disturbadas por el fuego y por la acción antrópica.

2. Alto grado de xerofitismo, sustentado en su capacidad de acceder a reservorios de agua profundos a través del sistema radicular profundo y en diversas estrategias fisiológicas (vía fotosintética $\mathrm{C}_{3}$ ), morfológicas y arquitecturales que le permiten evadir y tolerar la sequía (Delbón et al., 2010; Villagra et al., 2011).

3. Alta capacidad de rebrote o regeneración por semillas, por rebrote de las yemas existentes en el cuello de la planta y en las raíces (Martínez-Carretero y Dalmasso, 2002), vías de propagación que pueden darse en forma simultánea. La posesión de estructuras de regeneración vegetativa se asocia con la presencia en tiempo evolutivo de incendios recurrentes o períodos de sequía extensos (James, 1984; Keeley, 1992). Mientras que la división del tallo posibilita que parte de la planta sobreviva y se reproduzca en un microhábitat más favorable (Schenk, 1999), para la mayoría de estas especies la dispersión de las semillas se realiza por medio de los animales que consumen los frutos y dispersan las semillas con las deyecciones (Feldman, 2006).

4. Habilidad para fijar $\mathrm{N}$ atmosférico. Este atributo está presente en la mayoría de estas especies gracias a su simbiosis con bacterias nitrificantes, que unida a la condición de heliófilas hace que sean especies "pioneras" de la sucesión vegetal en ambientes disturbados (Zitzer, Archer y Boutton, 1996; Fredericksen, Justiniano, Mostacedo, Kennard y McDonald, 2000; Kunst, 2006).

5. Sistema radicular bien desarrollado y profundo (freatofismo), por lo que contribuyen a mantener la humedad del suelo y a disminuir el estrés hídrico en verano, a través del levantamiento hidráulico (Torres, Squeo, Jorquera, Aguirre y Ehleringer, 2002; León y Squeo, 2004), amortiguan el estrés ambiental debido al mayor aporte de los recursos superficiales -agua y nutrientes- (Meglioli, 2016).

6. Habilidad para colonizar ambientes. El efecto combinado de las características antes mencionadas les confiere una marcada superioridad competitiva frente a otros especímenes, a lo que se agrega que por ser mayoritariamente leguminosas cuentan con otras ventajas adicionales debido a que: i) poseen un capital inicial alto al momento de la instalación debido al mayor tamaño de las semillas (algunas son capaces de rebrotar en estado de plántula) asegurando la persistencia de los individuos que lograron instalarse; ii) producen más biomasa; y iii) destinan más biomasa por unidad de longitud de raíz que aquellas especies que no nodulan, sean deciduas o perennes (Barchuk et al., 2006).

7. Presencia de metabolitos secundarios en diversas partes y órganos de la planta, compuestos que las dotan de un incuestionable potencial nutracéutico (Rossi, 2003; Alonso, 2004; Barboza, Cantero, Núñez y Espinar, 2006; Rossi, De León, González y Pereyra, 2007; Guerrero, 2008; García, Aroguy y Nazareno, 2017; Villarreal, Lescano y Costa, 2017), y de un alto valor como fuentes de resinas y principios aromáticos (Amorín y Rosow, 1989).

Las características 0 atributos antes mencionados son responsables, en gran medida, de los impactos positivos o negativos que se les atribuyen a la mayoría de estas plantas, entre los que se destacaron:

1. Contribuyen a la protección del suelo ya que previenen la pérdida de nutrientes contrarrestando la erosión hídrica. El follaje y la hojarasca de los arbustos reducen la velocidad y, por consiguiente, la energía de las gotas que impactan el suelo (Gutiérrez y Squeo, 2004); mientras que el tronco y las raíces disminuyen la capacidad de los distintos agentes climáticos para transportar materiales (Espinoza,Fuentes y Molina, 1988). Reducen también el potencial erosivo del viento y contribuyen al ciclado de nutrientes (Gutiérrez y Squeo, 2004). Así, la remoción de la vegetación leñosa acelera dramáticamente los procesos degradativos, incrementándose el riesgo potencial de erosión hídrica (Magliano et al., 2016), el lavado de nutrientes y la desertificación en zonas áridas y semiáridas (Shachak,Sachs y Moshe, 1998).

2. Incrementan la diversidad faunística en el agroecosistema. Los arbustos tienen la capacidad de modificar el ambiente creando microclimas marcadamente diferentes de los presentes en espacios abiertos, por lo que devienen refugio ideal para la avifauna silvestre actuando como corredores biológicos, y favorecen el incremento de la biota edáfica (Silberman et al., 2017). Aunque estudios realizados en las zonas áridas de Sudamérica sugieren una influencia relativamente escasa sobre la riqueza específica del estrato herbáceo (Gutiérrez et al., 1993; Rossi y Villagra, 2003; Larrea-Alcázar, López y Barrientos, 2006), otros autores sostienen que, por el contrario, desempeñan un papel importante en este 
sentido (Gutiérrez y Squeo, 2004; López y Ortuño, 2008). Por esta razón han sido considerados ingenieros del ecosistema (Jones, Lawton y Sachak, 1997), propiciando un efecto nodriza e interacciones de facilitación en los ecosistemas (Bruno, Stachowicz y Bertness, 2003).

3. Las hojas, flores, frutos, tallos tiernos y hojarasca de muchas de estas especies resultan una biomasa comestible de alto valor nutritivo para el ganado, sobre todo en los períodos de escasez de forrajes provenientes del estrato graminoso (Rossi, 2003; Díaz, 2009; Pons, 2015).

4. Pueden constituir una fuente adicional de ingresos mediante la extracción, procesamiento y comercialización de madera, leña, carbón, briquetas, energía, extractos medicinales (Rossi, 2003; Alonso, 2004; Barboza et al., 2006; Rossi et al., 2007; Trillo y Demaio, 2007; Guerrero, 2008; García et al., 2017; Villarreal et al., 2017) y material tintóreo (Joseau, Meehan, Boboney Hernández, 2018), productos apícolas asociados (Demaio, Karliny Medina, 2015; Pons, 2015; Verzino et al., 2016; Palacio, Carrizo, Roger, Coria y Epstein, 2017).

5. A elevadas densidades reemplazan las pasturas o pastizales, consecuentemente, disminuyen la oferta forrajera proveniente del estrato graminoso, y reducen la receptividad o productividad del pastizal (Kunst, 2006; Rossi, 2006; Graz, 2008; Tighe, Reid, Wilson y Briggs, 2009). Este impacto negativo está relacionado con su habilidad para colonizar ambientes disturbados.

6. Dificultan el manejo de las pasturas y de los animales, así como el arreo del ganado (Silberman et al., 2017), provocan daños a los animales e interfieren negativamente con las actividades humanas en tiempo y espacio
(Feldman, 1976), dado por la presencia de espinas y su crecimiento enmarañado.

7. Disminuyen la accesibilidad de los animales al pastizal, así como la transitabilidad y visibilidad del ganado (Kunst, 2006; Rossi, 2006; Graz, 2008; Tighe et al., 2009; Silberman et al., 2017).

8. Afectan la rentabilidad del emprendimiento ganadero (Kunst, 2006; Kunst et al., 2016), ya sea en sentido negativo o positivo, dependiendo del componente animal predominante y del propósito del sistema productivo en cuestión. En este sentido, Rossi (2006) sostiene que cuando la producción y utilización del forraje baja, la producción de carne vacuna lo hace en una proporción variable según diferentes factores y, por consiguiente, los ingresos económicos del emprendimiento se ven disminuidos también.

En la Tabla 2 se exponen los valores medios modales, y la frecuencia relativa de aparición de cada uno de los impactos antes señalados, cuando son comparados en pares con los restantes por los productores ganaderos involucrados en la investigación. El coeficiente de variación correspondiente a cada media es superior al $40 \%$ en el $62,5 \%$ del total de valores, por lo que se infiere que existe una elevada variabilidad en la percepción que los entrevistados tuvieron sobre la importancia de los impactos, particularmente el referido a los ingresos provenientes de la comercialización de los derivados arbustivos. Esto podría estar relacionado con el grado de especialización de los productores y su orientación como sistema productivo hacia la subsistencia o la comercialización de la carne vacuna como renglón mayoritario.

La Tabla 3 contiene los valores medios y la frecuencia relativa de aparición de los impactos, según la importancia otorgada a cada uno por los técnicos y profesionales. Este sector ocupacional fue agrupado según el enfoque

Tabla 2. Frecuencia media, modal y relativa de aparición de los impactos atribuidos a las especies arbustivas, según la percepción de los productores $(n=53)$.

\begin{tabular}{lccccc}
\hline Efectos & Frecuencia media & EE & CV (\%) & Frecuencia modal & Frecuencia relativa de aparición \\
\hline Colonización & 5,09 & 0,24 & 34,5 & 7 & 0,73 \\
Protección & 3,11 & 0,27 & 62,9 & 3 & 0,44 \\
Diversidad & 1,64 & 0,15 & 66,6 & 1 & 0,23 \\
Alimentación & 3,25 & 0,26 & 57,3 & 5 & 0,46 \\
Manejo & 4,58 & 0,20 & 32,4 & 3 & 0,65 \\
Accesibilidad & 4,34 & 0,16 & 26,7 & 4 & 0,62 \\
Ingresos & 1,34 & 0,26 & 140,4 & 0 & 0,19 \\
Rentabilidad & 4,64 & 0,26 & 40,8 & 2 & 0,66 \\
\hline
\end{tabular}

Referencias: $\mathrm{EE}$ = Error estándar, CV = Coeficiente de variación 
productivista o conservacionista manifestado en las respuestas de los entrevistados. Así, para los "productivistas" (47,6 \% de los entrevistados), los impactos negativos relacionados con el manejo, la rentabilidad, la colonización y la accesibilidad fueron los de mayor importancia, mientras que para los "conservacionistas" $(52,4 \%)$ fueron los impactos positivos vinculados a la protección, la diversidad y la alimentación, unido al manejo como impacto negativo. Estos resultados confirman la complejidad del tema objeto de estudio y la diversidad de percepciones existentes entre los actores sociales vinculados al mismo y la literatura científica consultada.

Los resultados también sugieren que las especies arbustivas, lejos de constituir un tema polémico o discordante, exigen un análisis inter(trans)disciplinario e interinstitucional (Mettrick, 1993; Selener, 1997; Méndez y Overpeck, 2000; Méndez y Gliessman, 2002), donde la diversidad de criterios o posiciones filosóficas no conduzcan a la exclusión o marginación de actores sociales, sino al logro de un enfoque holístico y consensuado de todas las partes interesadas. Solo así podría alcanzarse un entendimiento común de las complejas realidades que caracterizan los agroecosistemas ganaderos del noroeste de Córdoba.

En estos agroecosistemas es muy recurrente el empleo del rolo y la rastra para el control mecánico de las especies arbustivas presentes en el estrato herbáceo (Bonfonte, 2006; Lizzi, 2006), y la aplicación de productos químicos o "arbusticidas" (Feldman, 2006; Bertín y Cepeda, 2007). Existen además recomendaciones para el uso del fuego prescripto o controlado (Güiraldes, 2006; Kunst et al., 2013; Kunst et al., 2015). Estos procedimientos también han sido utilizados en otras regiones del mundo (Masters y Sheley, 2001; Van Wilgen, Richardson y Higgins, 2001; Vitelli y Pitt, 2006), no existiendo coincidencia en cuanto a lo reportado sobre la eficiencia y eficacia de los mismos. Particularmente en el área de estudio, ninguno de ellos-empleados de manera individual o combinada- ha logrado el resultado esperado por los productores, disminuyendo cada vez más el intervalo entre intervenciones sucesivas, mientras que el renoval manifiesta mayor vigor y capacidad de colonización. Lo anterior queda corroborado con los valores de viabilidad contenidos en la Tabla 4, según la percepción de los entrevistados. Hay que tener en cuenta que no todos los actores sociales tienen experiencias o conocimientos sobre todos los tratamientos evaluados, razón por la cual el porcentaje de informantes descendió desde 83,3 y $80,9 \%$ (rolado y rastra) hasta 9,5\% para la combinación de rolado con fitocida (arbusticida) y fuego, respecto al número total de personas que participaron en la aplicación de la herramienta $(n=42)$.

Al considerar tanto el puntaje medio como la viabilidad relativa, se infiere que ninguno de los tratamientos alcanzó valores superiores a 9,7 puntos (de un máximo posible de 16) y 0,6 respectivamente, por tanto, no son totalmente viables y, en adición, incrementan significativamente los costos de producción lo cual coincide con lo apuntado por Lizzi (2006). Pretender la erradicación total de los renovales es antieconómico ya que supondría realizar intervenciones prácticamente todos los años (Rossi y Kunst, 2006) con el consiguiente impacto negativo sobre los recursos naturales contenidos en el agroecosistema.

Los ocho criterios utilizados para evaluar la viabilidad mostraron coeficientes de variación disímiles y en algunos casos elevados, por lo que

Tabla 3. Frecuencia media y relativa de aparición de los impactos atribuidos a las especies arbustivas, según la percepción de los técnicos y profesionales $(n=42)$.

\begin{tabular}{lcccccccc}
\hline \multirow{2}{*}{ Efectos } & \multicolumn{3}{c}{ Productivistas (n= 20) } & \multicolumn{3}{c}{ Conservacionistas (n= 22) } \\
\cline { 2 - 10 } & $\begin{array}{c}\text { Frecuencia } \\
\text { media }\end{array}$ & $\mathbf{E E}$ & $\begin{array}{c}\text { CV } \\
\mathbf{( \% )}\end{array}$ & $\begin{array}{c}\text { Frecuencia } \\
\text { relativa }\end{array}$ & $\begin{array}{c}\text { Frecuencia } \\
\text { media }\end{array}$ & EE & $\begin{array}{c}\text { CV } \\
\text { (\%) }\end{array}$ & $\begin{array}{c}\text { Frecuencia } \\
\text { relativa }\end{array}$ \\
\hline Colonización & 4,10 & 0,49 & 53,6 & 0,59 & 2,50 & 0,33 & 61,4 & 0,36 \\
Protección & 2,25 & 0,30 & 59,2 & 0,32 & 5,41 & 0,28 & 24,0 & 0,77 \\
Diversidad & 2,35 & 0,34 & 65,2 & 0,34 & 5,05 & 0,33 & 30,3 & 0,72 \\
Alimentación & 2,35 & 0,30 & 57,4 & 0,34 & 4,77 & 0,34 & 33,6 & 0,68 \\
Manejo & 5,10 & 0,35 & 30,4 & 0,73 & 3,05 & 0,35 & 54,1 & 0,44 \\
Accesibilidad & 5,05 & 0,26 & 22,7 & 0,72 & 2,18 & 0,36 & 78,3 & 0,31 \\
Ingresos & 1,80 & 0,38 & 94,7 & 0,26 & 3,32 & 0,32 & 45,9 & 0,47 \\
Rentabilidad & 5,00 & 0,26 & 23,4 & 0,71 & 1,73 & 0,31 & 84,2 & 0,25 \\
\hline
\end{tabular}

Referencias: $\mathrm{EE}=$ Error estándar, $\mathrm{CV}=$ Coeficiente de variación 
por sí solos no ofrecen un nivel de confiabilidad apropiado. El análisis combinado de los mismos aumenta significativamente el grado de certidumbre de la evaluación.

Contrario a lo que sostienen Janak y Davis (1997, citados por Lizzi, 2006), en general los tratamientos combinados no suelen mostrar efectos sinérgicos y no exhiben valores de viabilidad elevados, al menos para las experiencias vivenciales de los productores entrevistados. Estos resultados confirman la necesidad de transitar hacia enfoques que propicien el manejo agroecológico de estas especies arbustivas, en lugar de incrementar la frecuencia y severidad de los tratamientos convencionales. De modo que el reto presente y futuro es aprender a convivir con el renoval (Güiraldes, 2006) aprovechando sus bondades y minimizando sus impactos negativos.

La Tabla 5 presenta una breve descripción de las opciones identificadas, que fueron sometidas a un proceso de evaluación de factibilidad ex-ante, cuyos resultados se exponen en la Tabla 6.

Obsérvese que la mayoría de las opciones (83,3 \%) obtuvo más del $50 \%$ del valor máximo posible a alcanzar, de acuerdo a los presupuestos metodológicos de la herramienta aplicada, lo cual denota que existen amplias oportunidades para que sean implementadas en la práctica productiva del noroeste de Córdoba o en otras regiones de Argentina. No obstante, la correcta selección y apropiada combinación entre ellas son premisas que condicionarán el éxito deseado.

La opción referida al pago por servicios ambientales, con la mayor puntuación, es un mecanismo directo de conservación en el que los proveedores de servicios ecosistémicos reciben un pago por los servicios ambientales generados en sus predios (Gobbi, 2011); sin embargo, solo devendrá un incentivo de participación si los montos de pago a recibir por los proveedores compensan,

Tabla 4. Puntajes medio y modal, y viabilidad relativa de los tratamientos empleados para controlar las especies arbustivas.

\begin{tabular}{|c|c|c|c|c|c|c|c|c|}
\hline \multirow{2}{*}{ Descriptores } & \multirow{2}{*}{ Rolado } & \multirow{2}{*}{ Rastra } & \multirow{2}{*}{ Fitocida } & \multirow{2}{*}{ Fuego } & \multicolumn{4}{|c|}{ Combinaciones } \\
\hline & & & & & $\mathrm{Ro}+\mathrm{Fi}$ & $\mathrm{Ro}+\mathrm{Fu}$ & $\mathrm{Ro}+\mathrm{Ra}$ & $\mathrm{Ro}+\mathrm{Fi}+\mathrm{Fu}$ \\
\hline Puntaje medio & 9,71 & 9,63 & 8,52 & 9,30 & 9,40 & 8,00 & 9,53 & 5,50 \\
\hline $\mathrm{EE}$ & 0,47 & 0,43 & 0,60 & 0,40 & 0,88 & 1,26 & 0,78 & 0,96 \\
\hline CV $(\%)$ & 28,2 & 26,6 & 33,5 & 19,5 & 29,8 & 35,4 & 31,7 & 34,8 \\
\hline $\mathrm{N}$ & 34 & 35 & 23 & 20 & 10 & 5 & 15 & 4 \\
\hline Viabilidad relativa & 0,61 & 0,60 & 0,53 & 0,58 & 0,59 & 0,50 & 0,60 & 0,34 \\
\hline$\%$ informantes del total & 80,9 & 83,3 & 54,8 & 47,6 & 23,8 & 11,9 & 35,7 & 9,5 \\
\hline Moda & 11 & 11 & 9 & 10 & 9 & 5 & 10 & 4 \\
\hline
\end{tabular}

Referencias: $\mathrm{EE}=$ Error estándar, $\mathrm{CV}=$ Coeficiente de variación, $\mathrm{n}=$ Tamaño de la muestra, Ro+Fi = Rolo combinado con fitocida, $\mathrm{Ro}+\mathrm{Fu}=$ Rolo combinado con fuego, $\mathrm{Ro}+\mathrm{Ra}=$ Rolo combinado con rastra, $\mathrm{Ro}+\mathrm{Fi}+\mathrm{Fu}=$ Combinación de rolo con fitocida y fuego

Tabla 5. Descripción de las alternativas identificadas para propiciar el manejo agroecológico de las especies arbustivas.

\begin{tabular}{cl}
\hline Opción N. & \multicolumn{1}{c}{ Breve descripción de las opciones identificadas } \\
\hline $\mathbf{1}$ & $\begin{array}{l}\text { Rolado selectivo de baja intensidad con siembra de pasturas y plantación de especies arbóreas } \\
\text { nativas, de manera simultánea, en un diseño espacial silvopastoril y con protección de los plantines } \\
\text { contra herbivoría. }\end{array}$ \\
& $\begin{array}{l}\text { Uso de rastra con siembra de pasturas y plantación de especies arbóreas nativas, de manera simultánea, } \\
\text { en un diseño espacial silvopastoril y con protección de los plantines contra herbivoría. }\end{array}$ \\
& $\begin{array}{l}\text { Pastoreo rotativo secuencial: Se realizaría un pastoreo combinado, donde los bovinos realizarán la función } \\
\text { de "despuntadores" y los caprinos serán "continuadoreso repasadores" propiciando la defoliación de }\end{array}$ \\
& los arbustos y sus renovales. Implica la construcción de potreros o piquetes para la rotación. \\
& Comercialización de productos derivados de las arbustivas: Implicaría la extracción, procesamiento y \\
& venta de madera, leña, carbón, briquetas, energía, extractos medicinales, material tintóreo, productos \\
apícolas, etc. & $\begin{array}{l}\text { Poda de elevación de copa con raleo selectivo de arbustos: Consiste en despejar de ramas basales el } \\
\text { eje principal, hasta la altura de ramificación deseada para favorecer la accesibilidad y transitabilidad } \\
\text { del ganado, y el crecimiento y disponibilidad de las pasturas. } \\
\text { Conservación activa con retribución por servicio ambiental: Los productores recibirían un pago } \\
\text { compensatorio o reducción de la tasa impositiva por uso de la tierra, equivalente al ingreso neto } \\
\text { potencial mínimo por hectárea bajo conservación, sin intervención para fines productivos (ganadería). }\end{array}$ \\
$\mathbf{5}$ &
\end{tabular}


Tabla 6. Puntajes medio y modal, y factibilidad relativa de las alternativas agroecológicas evaluadas, según la percepción de los actores sociales.

\begin{tabular}{lcccccc}
\hline & \multicolumn{7}{c}{ Opciones numeradas en Tabla 5 } \\
\cline { 2 - 7 } & Ro+P+AN (1) & Ra+P+AN (2) & PRS-V+C (3) & ComDerArbus (4) & Poda (5) & PagoComp (6) \\
\hline Valor medio & 8,21 & 6,91 & 8,49 & 8,30 & 9,16 & 9,84 \\
EE & 0,38 & 0,42 & 0,33 & 0,33 & 0,30 & 0,33 \\
CV (\%) & 33,8 & 43,9 & 30,9 & 31,4 & 25,9 & 26,4 \\
N & 53 & 53 & 63 & 63 & 63 & 63 \\
Factibilidad relativa & 0,59 & 0,49 & 0,61 & 0,59 & 0,65 & 0,70 \\
Informantes del total (\%) & 84,1 & 84,1 & 100,0 & 100,0 & 100,0 & 100,0 \\
Moda & 9 & 9 & 9 & 11 & 10 & 10 \\
\hline
\end{tabular}

Referencias: $\mathrm{EE}=$ Error estándar, $\mathrm{CV}$ = Coeficiente de variación, $\mathrm{n}$ = Tamaño de la muestra

como mínimo, el costo de conservación y el costo de oportunidad del uso de la tierra resignado. En los últimos años se han desarrollado diversos mecanismos de pago en Bolivia (Wunder, 2008), Colombia (Echaverría, 2002), Costa Rica (Pagiola, 2008), El Salvador (Díaz, Dimas,García, Herrador y Méndez, 2002), México (Corbera, González y Brown, 2008), Ecuador (Wunder y Albán, 2008), cuyas experiencias positivas deberían tenerse en cuenta para realizar adecuaciones a la Ley Nacional 26.331 de Presupuestos Mínimos de Conservación de Bosques Nativos y otros marcos regulatorios, de manera tal que el pago monetario o reducción de la tasa impositiva por uso de la tierra se equipare al ingreso neto potencial mínimo por hectárea bajo conservación, sin la realización de intervenciones más allá de las recomendadas para promover la conservación activa y el manejo forestal sustentable. En este sentido, resulta imprescindible desarrollar un proceso participativo de manera que todas las partes interesadas puedan expresar sus puntos de vista y estos sean tenidos en cuenta por los tomadores de decisiones pertenecientes al poder ejecutivo provincial y nacional.

La poda de elevación de copa, opción ubicada en el segundo lugar de preferencia, es una práctica silvicultural bien conocida en arboricultura (Ledesma, 2008; Purcell, 2013; Remilo, 2015) y es aplicable a los arbustos presentes en los agroecosistemas del noroeste de Córdoba para minimizar los impactos negativos de estos relacionados con la accesibilidad y transitabilidad de los animales y de los ganaderos, y el crecimiento de las pasturas.

Las ventajas del pastoreo rotativo o rotacional son bien conocidas (Díaz, 2009) y la combinación secuencial de vacunos y caprinos, especies con hábitos alimentarios diferentes (pastoreo vs. ramoneo), posibilita una mayor intensidad de defoliación de las especies arbustivas con el consiguiente control biológico que ejercen los caprinos (Luginbuhl y Pietrosemoli, 2007; Yayneshet, Eik y Moe, 2008) sobre estas plantas y sus renovales, por tanto se infiere que estos argumentos justifican el hecho que esta opción se ubique entre las tres más factibles según la percepción de los entrevistados.

La extracción, procesamiento y comercialización de los productos derivados de las arbustivas (madera, leña, carbón, briquetas, energía, extractos medicinales, material tintóreo, productos apícolas, etc.) es la cuarta opción con mayor puntaje medio y factibilidad relativa. Los atributos beneficiosos de estas plantas son reconocidos por los productores, técnicos y especialistas, a pesar de que no son abundantes las experiencias exitosas publicadas al respecto. Ello sería una importante contribución para diversificar los ingresos y reforzar el carácter multifuncional de los sistemas productivos.

Las opciones relacionadas con el uso del rolo selectivo de baja intensidad y la rastra, combinadas con la siembra simultánea de pasturas son prácticas conocidas por los productores e implican, en primer lugar, el cumplimiento de las regulaciones y normativas ambientales vigentes, así como la elaboración de un diseño o arreglo espacial silvopastoril (Gallego, Morales y Vivas, 2012; Reinoso, 2013). La novedad de estas opciones consiste en la plantación de especies arbóreas nativas para lo cual es necesaria la protección de los plantines contra herbivoría con la consiguiente prolongación del período de clausura que tradicionalmente realizan los productores. El incremento de la presencia de árboles nativos en estos agroecosistemas, dentro de niveles que no superen el 40 \% (Díaz, 2003; 2009), desempeñaría un papel regulador de los estratos vegetales inferiores (Díaz, 1992), de manera tal que se favorezca el crecimiento de la cobertura herbácea de interés forrajero y el mantenimiento de niveles deseables del componente arbustivo. 


\section{CONCLUSIONES}

La presencia de múltiples atributos beneficiosos en estas especies (provisión de follaje y frutos comestibles; flores melíferas, madera para obra y combustibles, fibra, extractos medicinales, entre otros) propicia la implementación de estrategias de manejo y uso productivo en sistemas agroforestales multifuncionales. El pago por servicios ambientales, la poda de elevación de copa, el pastoreo rotativo secuencial vacuno-caprino, y la comercialización de los productos derivados de estas plantas, son las alternativas ecoamigables más promisorias.

\section{AGRADECIMIENTOS}

Los autores agradecen al Centro Latinoamericano de Formación Interdisciplinaria Sustentabilidad y Desarrollo dependiente de la Secretaría de Ciencia y Tecnología de la UNC y del Ministerio de Ciencia, Tecnología e Innovación Productiva (Argentina) y a la Facultad de Ciencias Agropecuarias UNC por el apoyo humano y financiero otorgado para la realización de la presente investigación.

\section{BIBLIOGRAFÍA}

Adema, E. O., Buschiazzo, D. E., Babinec, F. J., Rucci, T. E. y Gómez-Hermida, V. F. (2004). Mechanical control of shrubs in the semiarid region of Argentina and its effects on soil water content and grassland productivity. Agricultural Water Management, 68, 185194. doi: https://doi.org/10.1016/j.agwat.2004.04.001.

Alonso, J. R. (2004). Tratado de Fitofármacos y Nutracéuticos. Buenos Aires, Argentina: Ed. Corpus.

Amorín, J. L. y Rosow, R. A. (1989). Guía taxonómica con plantas de interés farmacéutico (Segunda edición). Dominguezia,7 (1), 31-38.

Archer, S. (1995). Tree-grass interactions in a Prosopisthornscrub savanna parkland: reconstructing the past and predicting the future. Ecoscience, 2, 83-89. doi:10 .1080/11956860.1995.11682272.

Barboza, G. E., Cantero, J. J., Núñez, C. O. y Espinar, L. A. (2006). Flora Medicinal de la provincia de Córdoba (Argentina). Pteridófitas y Antófitas silvestres o naturalizadas. Córdoba, Argentina: Ed. Museo Botánico de Córdoba.

Barchuk, A. H., Campos, E. B., Oviedo, C. y Díaz, M. P. (2006). Supervivencia y crecimiento de plántulas de especies leñosas del Chaco Árido sometidas a remoción de la biomasa aérea. Ecología Austral, 16, 47-61.
Bertín, O. y Cepeda, S. (2007). Defoliación y control químico de duraznillo blanco (Solanum glaucophyllum) en pastizales naturales. Revista Argentina de Producción Animal, 27 (2), 67-74.

Bond, W. J. y Midgley, J. J. (2001). Ecology of sprouting in woody plants: the persistence niche. Trends Ecol. Evol., 16, 45-51. doi: https://doi.org/ 10.1016/S01695347(00)02033-4.

Bonfonte, A. (2006). Control mecánico del renoval. En C. Kunst (Comp.) Manejo y control del renoval. Memorias de la Primera Jornada de Actualización Técnica en Manejo y Control del Renoval (159-162). Santiago del Estero, Argentina: Ediciones INTA.

Borrás, M., Manghi, E., Miñarro, F., Monaco, M., Navall, M., Peri, P., Periago M. E. y Preliasco, P. (2017). Acercando el Manejo de Bosques con Ganadería Integrada al monte chaqueño. Una herramienta para lograr una producción compatible con la conservación del bosque. Buenas prácticas para una ganadería sustentable. Kit de extensión para el Gran Chaco. Buenos Aires, Argentina: Fundación Vida Silvestre Argentina.

Bruno, J. F., Stachowicz, J. J. y Bertness, M. D. (2003). Inclusion of facilitation into ecological theory. TRENDS in Ecology and Evolution, 18 (3), 119-125. doi: 10.1016/ S0169-5347(02)00045-9.

Cabrera, A. L. (1994). Regiones Fitogeográficas Argentinas. En Kugler, W. F.(Ed.) Enciclopedia Argentina de Agricultura y Jardinería. Tomo // ( $2^{\mathrm{a}}$ ed.). Buenos Aires, Argentina: Editorial ACME.

Corbera, E., González, C. y Brown, K. (2008). Institutional dimension of payments for ecosystem services: an analysis of Mexico's carbon forestry program. Ecological Economics, 68, 743-761. doi: https://doi. org/10.1016/j.ecolecon.2008.06.008.

Daza, C. G. y Sánchez, C. (2009). Zonas Agroeconómicas Homogéneas Córdoba. Estudios socioeconómicos de la sustentabilidad de los sistemas de producción y recursos naturales (Ediciones INTA No. 10). Buenos Aires, Argentina: Instituto Nacional de Tecnología Agropecuaria.

Delbón, N., Cortez, M. A., Castello, L., Ríos, J. C. A., Villamil, M., Risso, R., Dottori, N. y Cosa, M. T. (2010). Anatomía foliar y estrategias adaptativas en especies arbustivas de las Sierras Chicas de Córdoba, Argentina. Arnaldoa, 17(1), 41-49.

Demaio, P .H., Karlin, U. O. y Medina, M. J. (2015). Árboles nativos de Argentina. Tomo I: Centro y Cuyo. Córdoba, Argentina: Ecoval Ediciones. Díaz, R. O. (1992). Evaluación de los recursos forrajeros del Chaco Árido. En Karlin, U. y Coirini, R. (Eds.). Sistemas agroforestales para pequeños productores de zonas áridas (18-23).Córdoba, Argentina: Facultad 
de Ciencias Agropecuarias, Universidad Nacional de Córdoba.

Díaz, R. O. (2003). Efectos de diferentes niveles de cobertura arbórea sobre la producción acumulada, digestibilidad y composición botánica del pastizal natural del Chaco Árido (Argentina). AgriScientia, 20, 61-68. doi:http://dx.doi.org/10.31047/1668.298x.v20. n0.2832.

Díaz, R. O. (2009). Utilización de pastizales naturales (edición revisada). Córdoba, Argentina: Encuentro Grupo Editor.

Díaz, O., Dimas, L., García, M., Herrador, L. y Méndez, V. (2002). Pago por servicios ambientales en El Salvador. San Salvador, El Salvador: PRISMA.

Di Rienzo, J., Casanoves, F., González, L.,Tablada, M., Robledo, C. W. y Balzarini, M. (2017). Infostat (Versión 2017). [Software estadístico]. Córdoba, Argentina: Facultad de Ciencias Agropecuarias, Universidad Nacional de Córdoba.

Echaverría, M. (2002). Water user associations in the Cauca Valley: A voluntary mechanism to promote upstream-downstream cooperation in the protection of rural watersheds. Land-Water Linkages in Rural Watershed Study Series. Roma, Italia: FAO.

Espinoza, G. A., Fuentes, E. R. y Molina, J. D. (1988). La erosión: fenómenos naturales y acción del hombre. En E. R. Fuentes y S. Prenafeta (Eds.), Ecología del Paisaje en Chile Central. Santiago, Chile: Ediciones Universidad Católica de Chile.

Feldman, I. (1976). Consideraciones acerca de una definición de malezas leñosas. Aproximación para su solución. Resúmenes VII Reunión Argentina de Malezas y su control (pp. 60-61).Buenos Aires, Argentina.

Feldman, I. (2006). Control químico del renoval. En Kunst,C. (Comp.), Manejo y control del renoval. Memorias de la Primera Jornada de Actualización Técnica en Manejo y Control del Renoval (pp.115117). Santiago del Estero, Argentina: Ediciones INTA.

Fredericksen, T. S., Justiniano, M. J., Mostacedo, B., Kennard, D. y Mc Donald, L. (2000). Comparative regeneration ecology of three leguminous timber species in a Bolivian tropical dry forest. New Forests, 20, 45-64. doi:https://doi.org/10.1023/A:1006735819449.

Gallego, E. J., Morales, S. y Vivas, N. J. (2012). Propuesta para el uso de especies arbóreas y arbustivas forrajeras en sistemas ganaderos en el Valle del Patía, Cauca. Biotecnología en el Sector Agropecuario y Agroindustrial, 10(2), 207-216.

García, E. M., Aroguy, J. I. y Nazareno, M. A. (2017). La incorporación de especies leñosas en la dieta de cabrillas criollas (Capra hircus) permite obtener carne con mayor estabilidad oxidativa. En Albanesi, A. S., Domínguez, J. A., Helman, S., Nazareno M. y
Rodríguez, S. (Eds.), Aportes de la FAyA para el desarrollo agropecuario y agroindustrial del NOA. Tomo II. Cadena de valor en sistemas pecuarios y otros trabajos (1-13). Santiago del Estero, Argentina: Universidad Nacional de Santiago del Estero.

Geilfus, F. (2002). 80 herramientas para el desarrollo participativo: diagnóstico, planificación, monitoreo, evaluación. San José, Costa Rica: Instituto Interamericano de Cooperación para la Agricultura (IICA).

Giorgisi, M. A. y Tecco, P. A. (2014). Árboles y arbustos invasores de la Provincia de Córdoba (Argentina): una contribución a la sistematización de bases de datos globales. Bol. Soc. Argent. Bot., 49 (4), 581-603.

Gobbi, J. A. (2011). Pago por Servicios Ambientales: ¿Qué Son y Cómo Funcionan? En P. Laterra, E. G. Jobbágy, J. M. Paruelo (Eds.), Valoración de servicios ecosistémicos: conceptos, herramientas y aplicaciones para elordenamiento territorial (293-311). Buenos Aires, Argentina: Ediciones INTA.

Graz, F. (2008). The woody weed encroachment puzzle: gathering pieces. Ecohydrology, 1, 340-348. doi: https://doi.org/10.1002/eco.28

Guerrero, N. (2008). Uso y valoración de plantas medicinales y tintóreas presentes en Santiago del Estero, Argentina. Trabajo Fin de Grado, Universidad Politécnica de Madrid, Madrid, España. Recuperado de: http://oa.upm.es/1299/

Güiraldes, A. (2006). El fuego: una herramienta más. En Kunst C. (Comp.) Manejo y control del renoval. Memorias de la Primera Jornada de Actualización Técnica en Manejo y Control del Renoval (97-103). Santiago del Estero, Argentina: Ediciones INTA.

Gutiérrez, J. R. y Squeo, F. A. (2004). Importancia de los arbustos en los ecosistemas semiáridos de Chile. Ecosistemas, 13 (1), 36-45. Recuperado de: http://hdl. handle.net/10045/8801

Gutiérrez, J. R., Meserve, P. L., Jaksic, J. M., Contreras, L. C., Herrera, S. y Vásquez, H. (1993). Structure and dynamics of vegetation in a Chilean semiarid thornscrub community. Acta Oecólogica, 14, 271-285.

James, S. (1984). Lignotubers and burls. Their structure, function and ecological significance in Mediterranean ecosystems. Bot. Rev., 50, 225-266. doi: https://doi. org/10.1007/BF02862633.

Jones, C. G.,Lawton, J. H. y Sachak, M. (1997). Positive and negative effects of organisms as physical ecosystem engineers. Ecology,78(7), 1946-1957. doi: http://www.jstor.org/stable/2265935.

Joseau, M. J., Meehan, A. R., Bobone, A. y Hernández, R. (2018). El uso múltiple del bosque. Producción sostenible de fibras, tejidos y tintes naturales en Argentina. Roma, Italia: Istituto Superiore per la Protezione e la Ricerca Ambientale (ISPRA). 
Recuperado de:http://www.isprambiente.gov.it/it/ pubblicazioni/manuali-e-linee-guida/multifunzionalitadel-bosco.-produzione-sostenibile-di-fibre-tessuti-etinture-naturali-in-argentina.

Keeley, J. (1992). Recruitment of seedling and vegetative sprouts in unburned chaparral. Ecology, 73(4), 11941208. doi: https://doi.org/10.2307/1940669.

Kunst, C. (2006). El renoval: pasado, presente y futuro. En Kunst C. (Comp.) Manejo y control del renoval. Memorias de la Primera Jornada de Actualización Técnica en Manejo y Control del Renoval (9-14). Santiago del Estero, Argentina: Ediciones INTA.

Kunst, C., Bravo, S., Moscovich, F., Herrera, J., Godoy, J. y Vélez, S. (2003). Fecha de aplicación de fuego y diversidad de herbáceas en una sabana de Elionorus muticus (Spreng) O. Kuntze. Rev. Chil. Hist. Nat., 76(1), 105-115. doi: http://dx.doi.org/10.4067/S0716078X2003000100010.

Kunst, C., Ledesma, R., Bravo, S., Albanesi, A., Anriquez, A., Van Meer, H. y Godoy, J. (2013). Disrupting woody steady states in the Chaco region (Argentina): responses to combined disturbance treatments. Ecological Engineering, 42, 42-53.

Kunst, C., Ledesma, R., Bravo, S., Defossé, G., Godoy, J., Navarrete, V. y Jaime, N. (2015). Dinámica del contenido de humedad de pastos y su relación con la ecología del fuego en región chaqueña occidental (Argentina). Revista Investigaciones Agropecuarias, 41, 83-93.

Kunst, C., Navall, M., Ledesma, R., Silberman, J., Anriquez, A., Coria, D., Bravo, S., Gómez, A., Albanesi, A., Grasso, D., Domínguez-Núñez, J., González, A., Tomsic, P. y Godoy, J. (2016). Silvopastoral Systems in the western Chaco region, Argentina. En Peri, P., Dube, F. y Varella, A. (Eds.), Silvopastoral Systemas in Southern South America. Advances in Agroforestry 11. Basel, Suiza: Springer.

Larrea-Alcázar, D., López, R. P. y Barrientos, D. (2006). The nurse plant effect of Prosopis flexuosa DC (Leg. Mim.) in a dry valley of the Bolivian Andes. Ecotrópicos, 18(2), 89-95.

Ledesma, M. (2008). Arbolado público. Conceptos. Manejo. Estación Experimental Agropecuaria Manfredi, Córdoba, Argentina: Ediciones INTA.

Ledesma, R. y Tomsic, P. (Comp.) (2011). Manejo y control del renoval. Memorias de la Segunda Jornada de Actualización Técnica en Manejo y Control del Renoval. Santiago del Estero, Argentina: Ediciones INTA.

León, M. F. y Squeo, F. A. (2004). Levantamiento hidráulico: la raíz del asunto. En Cabrera, H. M.(Ed.), Ecofisiología de Plantas (99-109). Valparaíso, Chile: Ediciones Pontificia Universidad Católica de Valparaíso.
Ley de Presupuestos Mínimos de Conservación de Bosques Nativos. (2007, 28 de noviembre). Boletín Oficial 31310, Diciembre 26, 2007.

López, R. P. y Ortuño, T. (2008). La influencia de los arbustos sobre la diversidad y abundancia de plantas herbáceas de la Prepuna a diferentes escalas espaciales. Ecología Austral, 18, 119-131.

Lizzi, J. M. (2006). Control mecánico del renoval: la experiencia del CREA Semiárido Norte. En Kunst, C. (Comp.) Manejo y control del renoval. Memorias de la Primera Jornada de Actualización Técnica en Manejo y Control del Renoval (163-172). Santiago del Estero, Argentina: Ediciones INTA.

Luginbuhl, J. M. y Pietrosemoli, S. (2007). Utilización de caprinos para el control de vegetación indeseable. Arch. Latinoam. Prod. Anim., 15(1), 294-309.

Magliano, P. N.,Fernández, R. J.,Giménez, R., Marchesini, V. A., Páez, R. A. y Jobbágy, E. G. (2016). Cambios en la partición de flujos de agua en el Chaco Árido al reemplazar bosques por pasturas. Ecol. Austral, 26 (2), 95-106.

Martínez-Carretero, E. y Dalmasso, A. (2002). Response to cutting of Larrea divaricata and L. cuneifolia in Argentina. Appl. Veget. Sci., 5, 127-133.

Meglioli, P. A. (2016). Efectos de los disturbios provocados por puestos ganaderos sobre el complejo agua-suelovegetación en ecosistemas áridos acoplados al acuífero freático. Ecosistemas, 25 (3), 149-153. doi: http://dx.doi.org/10.7818/ECOS.2016.25-3.20.

Masters, R. y Sheley, R. (2001). Invited Synthesis Paper: Principles and practices for managing rangeland invasive plants. Journal of Range Management, 54, 502-517.

Méndez, V. E. y Gliessman, S. R. (2002). Un enfoque interdisciplinario para la investigación en agroecología y desarrollo rural en el trópico latinoamericano. Manejo Integrado de Plagas y Agroecología (Costa Rica), 64, 5-16.

Méndez, V. E. y Overpeck, D. (2000). Participatory research approaches in natural resource management: literature review summary and annotated bibliography. Documento preparado para el Seminario de Postgrado en Gobernabilidad Ambiental, Universidad de California, Santa Cruz, Estados Unidos.

Mettrick, H. (1993). Development oriented Research in Agriculture. Wageningen, Países Bajos: International Centre for Development Oriented Research in Agriculture (ICRA).

Mussari, C. M. (2005). Las características productivas de la región NOA. Situación actual y potencial. I Congreso Nacional de Invernada. El negocio ganadero depende de todos nosotros. Rosario, Argentina: Ed. AACREA. 
Orellana, C. R. (2003). Raza Criolla Argentina: evaluación integral desde la perspectiva de la ganadería ecológica en el Noroeste de Argentina. Tesis doctoral no publicada. Universidad de Córdoba, Córdoba, España.

Pagiola, S. (2008). Payments for environmental services in Costa Rica. Ecological Economics, 65, 712-724. doi: http://dx.doi.org/10.1016/j.ecolecon.2007.07.033.

Palacio, M. O., Carrizo, E., Roger, E., Coria, O. y Epstein, M. F. (2017). Flora leñosa de interés apícola en bosques serranos de Choya y Guisayán, Santiago del Estero. En Albanesi, A.S., Domínguez, J. A., Helman, S., Nazareno M. y Rodríguez, S. (Eds.) Aportes de la FAyA para el desarrollo agropecuario y agroindustrial del NOA. Tomo II. Cadena de valor en sistemas pecuarios y otros trabajos (15-24). Santiago del Estero, Argentina: Universidad Nacional de Santiago del Estero.

Pons, S. M. (2015). Guía de reproducción de árboles nativos de Córdoba para jardinería. Córdoba, Argentina: SIMA Editora.

Purcell, L. (2013). Trees and Storms, FNR-FAQ-12-W. West Lafayette, Estados Unidos: Purdue University Cooperative Extension.

Reinoso, M. (2013). Agroforestry: A viable alternative for Sustainable Land Management. Proceedings of the II Scientific Conference on Desertification and Drought, organized by the United Nations Convention to Combat Desertification. Bonn, Alemania: UNCCD.

Remilo, D. I. (2015). Poda de Arbolado Público. Módulo 2. Aspectos técnicos de la Poda. Charla Informativa. Memorias de la Jornada de Capacitación para Municipios. Estación Forestal Parque Pereyra Iraola Vivero Carlos Darwin. Facultad de Ciencias Agrarias y Forestales, UN La Plata, Buenos Aires, Argentina.

Renolfi, R. (2006). Control de arbustos mediante Pastoreo con Caprinos en un Arbustal - Bosque Abierto de la Región Chaqueña Semiárida. En C. Kunst (Comp.) Manejo y control del renoval. Memorias de la Primera Jornada de Actualización Técnica en Manejo y Control del Renoval (129-140). Santiago del Estero, Argentina: Ediciones INTA.

Rossi, B. E. y Villagra, P.E. (2003). Effects of Prosopis flexuosa on soil properties and the spatial pattern of understorey species in arid Argentina. Journal of Vegetation Science, 114, 543-550. Recuperado de: https://www.jstor.org/stable/3236829

Rossi, C. A. (2003). Componentes antinutricionales de especies leñosas forrajeros de los pastizales del Chaco Árido Argentino. Tesis de Maestría no publicada. Universidad de Córdoba, Córdoba, Argentina.

Rossi, C. A., De León, M., González, G. L. y Pereyra, A.M. (2007). Presencia de metabolitos secundarios en el follaje de diez leñosas de ramoneo en el bosque xerofítico del Chaco Árido Argentino. Tropical and Subtropical Agroecosystems, 7, 133-143. Recuperado de: http://www.redalyc.org/articulo.oa?id=93970208

Rossi, D. (2006). El Renoval. Enfoque y Perspectivas del Sector Empresario. En C. Kunst (Comp.) Manejo y control del renoval. Memorias de la Primera Jornada de Actualización Técnica en Manejo y Control del Renoval (39-45). Santiago del Estero, Argentina: Ediciones INTA.

Rossi, D. y Kunst, C. (2006). El renoval y manejo de pasturas. En C. Kunst (Comp.) Manejo y control del renoval. Memorias de la Primera Jornada de Actualización Técnica en Manejo y Control del Renoval (173-178). Santiago del Estero, Argentina: Ediciones INTA.

Schenk, J. H. (1999). Clonal splitting in desert shrubs. Plant Ecology, 141, 41-52

Selener, D. (1997). Participatory action research and social change. Nueva York, Estados Unidos: Cornell University Press

Shachak, M., Sachs, M. y Moshe, I. (1998). Ecosystem management of desertified shrublands in Israel. Ecosystems, 1, 475-483. Recuperado de: http://www. jstor.org/stable/3658682

Silberman, J., Albanesi, A. S., Anriquez, A., DomínguezNúñez, J. A., Kunst, C. y Grasso, D. (2017). Rol de los Sistemas Silvopastoriles en la conservación de la materia orgánica y biodiversidad del suelo. En Albanesi, A. S., Domínguez, J. A., Helman, S., Nazareno, M. y Rodríguez, S. (Eds.), Aportes de la FAyA para el desarrollo agropecuario y agroindustrial del NOA. Tomo II. Cadena de valor en sistemas pecuarios y otros trabajos (37-59). Santiago del Estero, Argentina: Universidad Nacional de Santiago del Estero.

Skarpe, C. (1990). Shrub layer dynamics under different herbivore densities in an arid savanna, Botswana. J. Applied. Ecol., 27, 873-885. doi: http://dx.doi. org/10.2307/2404383.

Tighe, M., Reid, N., Wilson, B. y Briggs, S. (2009). Invasive native scrub and soil condition in semi-arid south-eastern Australia. Agriculture, Ecosystems y Environment, 132, 212-222. doi: http://dx.doi. org/10.1016/j.agee.2009.04.001

Tolozano, B., Pisani, J. M. y Puricelli, E. C. (2016). Control mecánico y químico de Tessaria dodoneifolia (Hook. et Arn.) Cabrera (chilca dulce).RIA. Rev. Investig. Agropecu., 43(2), 128-134. Recuperado de:http:// www.redalyc.org/articulo.oa?id=86452401008

Torres, R., Squeo, F. A., Jorquera, C., Aguirre, E. y Ehleringer, J. R. (2002). Evaluación de la capacidad estacional de utilizar eventos de precipitación en tres especies de arbustos nativos con distintos sistemas 
radiculares. Revista Chilena de Historia Natural, 75, 737-749. doi: http://dx.doi.org/10.4067/S0716078X2002000400009.

Trillo, C. y Demaio, P. (2007). Tintes naturales: Guía para el reconocimiento y uso de plantas tintóreas del centro de Argentina. Córdoba, Argentina: Ediciones SEZO.

Van Wilgen, B., Richardson, D. y Higgins, S. (2001). Integrated control of invasive alien plants in terrestrial ecosystems. Land Use and Water Resources Research, 1, 1-6. Recuperado de: http://ageconsearch.umn.edu/ record/47853/files/paper01-05.pdf

Verzino, G. E., Hernández, R. A., Meehan, A. R., Joseau, M. J., Osés, D. H., Frassoni, J., Sández, S., Clausen, G., Salgado, C. E., Sosa, E. E. y Cisternas, P. A. (2016). Flora del bosque nativo del centro de Argentina. Valor paisajístico, tintóreo y apícola. Córdoba, Argentina: Editorial Brujas.

Villagra, P. E., Giordano, C., Álvarez, J. A., Cavagnaro, J. B., Guevara, A., Sartor, C., Passera, C.B.y Greco, S. (2011). Ser planta en el desierto: estrategias de uso de agua y resistencia al estrés hídrico en el Monte Central de Argentina. Ecología Austral, 21, 29-42.

Villarreal, M. E., Lescano, N. E. y Costa, K. E. (2017). El desafío de incorporar frutos silvestres subexplotados del monte semiárido argentino a sistemas productivos rentables. EnA.S. Albanesi, J. A. Domínguez, S. Helman,M. Nazareno y S. Rodríguez (Eds.), Aportes de la FAyA para el desarrollo agropecuario y agroindustrial del NOA. Tomo II. Cadena de valor en sistemas pecuarios y otros trabajos (15-29). Santiago del Estero, Argentina: Universidad Nacional de Santiago del Estero.

Vitelli, J. y Pitt, J. (2006). Assessment of current weed control methods relevant to the management of the biodiversity of Australian rangelands. Rangeland Journal, 28, 37-46. doi: https://doi.org/10.1071/ RJ06016.

Wunder, S. (2008). Payments for environmental services and the poor: Concepts and preliminary evidence. Environment and Development Economics, 13(3), 279-297. doi: https://doi.org/10.1017/ S1355770X08004282.

Wunder, S. y Albán, M. (2008). Decentralized payments for environmental services: The cases of Pimampiro and PROFAFOR in Ecuador. Ecological Economics, 65(4), 685-698. doi: https://doi.org/10.1016/j. ecolecon.2007.11.004.

Yayneshet, T., Eik, L. O. y Moe, S. R. (2008). Influences of fallow age and season on the foraging behavior and diet selection pattern of goats (Capra hircus L.). Small Rumin. Res., 77, 25-37. doi: http://dx.doi.org/10.1016/j. smallrumres.2008.03.001.

Zitzer, S. F., Archer, S.R. y Boutton, T. W. (1996). Spatial variability in the potential for symbiotic $\mathrm{N}_{2}$ fixation by woody plants in a subtropical savanna ecosystem. J. Appl. Ecol., 33, 1125-1136. 